\title{
Use of Galerkin Technique to the Rolling of a Plate in Deep Water
}

\author{
Swagata Ray ${ }^{a}$, Soumen De ${ }^{a}$ and B. N. Mandal ${ }^{b}$ \\ ${ }^{a}$ Department of Applied Mathematics, University of Calcutta \\ 92, A.P.C. Road, Kolkata-700 009, India \\ ${ }^{b}$ Physics and Applied Mathematics Unit, Indian Statistical Institute \\ 203, B.T. Road, Kolkata-700 108, India \\ E-mail(corresp.): bnm2006@rediffmail.com \\ E-mail: ray.swagata.06@gmail.com \\ E-mail: soumenisi@gmail.com
}

Received May 20, 2020; revised January 8, 2021; accepted January 9, 2021

\begin{abstract}
The classical problems of surface water waves produced by small oscillations of a thin vertical plate partially immersed as well as submerged in deep water are reinvestigated here. Each problem is reduced to an integral equation involving horizontal component of velocity across the vertical line outside the plate. The integral equations are solved numerically using Galerkin approximation in terms of simple polynomials multiplied by an appropriate weight function whose form is dictated by the behaviour of the fluid velocity near the edge(s) of the plate. Fairly accurate numerical estimates for the amplitude of the radiated wave at infinity due to rolling and also for swaying of the pate in each case are obtained and these are depicted graphically against the wave number for various cases.
\end{abstract}

Keywords: rolling motion, partially immersed and submerged plate, integral equation, Galerkin approximation, amplitude of radiated wave.

AMS Subject Classification: $74 \mathrm{~F} 10$.

\section{Introduction}

Study of water wave problems wherein waves are produced by rolling motion of a partially immersed as well as a submerged plate which executes simple harmonic oscillations about a horizontal axis was considered by a number of researchers. The rolling motion of a ship modelled as a partially immersed thin

Copyright (C) 2021 The Author(s). Published by Vilnius Gediminas Technical University

This is an Open Access article distributed under the terms of the Creative Commons Attribution License (http://creativecommons.org/licenses/by/4.0/), which permits unrestricted use, distribution, and reproduction in any medium, provided the original author and source are credited. 
plate in infinitely deep water was first investigated by Ursell [31] who obtained the explicit expression for the amplitude at infinity of the wave motion. Later, Haskind [11] obtained the exact values for general coefficients of damping and mass coupling for partially immersed plate. Evans [6] used Green's integral theorem to derive a simple expression for the amplitude of the radiated waves at infinity for the same problem considered by Ursell [31] and also deduced the wave amplitude for swaying of the plate. Later, Mandal [19] also used Green's integral theorem to obtain the amplitude of the radiated wave produced by small oscillations of a thin vertical plate submerged in deep water and also considered the case of swaying of the plate. The two-dimensional problem of the small-amplitude forced rolling motion of a thin vertical plate in finite depth water was considered by Evans and Porter [9]. They used two complementary formulations in conjunction with an accurate and efficient Galerkin method to solve the problem. Later, Banerjea and Mandal [2] considered the generation of waves due to rolling of a thin vertical plate which was solved by Green's integral theorem.

The problem of the generation of waves due to small rolling oscillations of a thin vertical plate partially immersed in uniform finite-depth water is investigated by Banerjea et al. [1] using eigenfunction expansion of the velocity potentials and also by hypersingular integral equation formulation. Mandal et al. [20] used dual integral equations involving trigonometric functions as kernel to reinvestigate the classical rolling ship problem due to small rolling oscillations of a thin vertical plate partially immersed in deep water. Later, Rakshit and Banerjea [26] investigated the effect of a small bottom undulation of the sea bed on the surface waves generated due to a rolling oscillation of a vertical plate either partially immersed or completely submerged in water of variable depth using Green's integral theorem.

The Galerkin approximation is a useful method to obtain an approximate solution of integral equations. The advantage of this method is that the set of functions need not be an orthogonal set or not necessary be complete. The Galerkin method converges faster than the collocation method (cf. Kanoria and Mandal [13]). Previously, Evans and Morris [8], Porter and Evans [25] employed Galerkins approximation technique followed by the Havelocks [12] expansion of water wave potential to obtain numerical estimates of reflection coefficient for oblique incidence of the wave train on a single vertical barrier in deep as well as finite depth water. Evans and Fernyhough [7] investigated about the numerical evidence of the existence of edge waves travelling along a periodic coastline using Galerkin approximation technique.

The water wave scattering by vertical thin porous barriers has been solved by Li et al. [15] considering a surface piercing barrier and a submerged bottomstanding barrier using a multi-term Galerkin method. In recent times, Meng et al. [24] presented a hybrid element-free Galerkin (HEFG) method for solving three dimensional wave propagation problem. Also Liu and Cheng [16], Liu et al. [17] and Cheng et al. [3] employed element-free Galerkin method in the study of some problems involving swelling of polymer gels, elastoplastic deformation problems and 3D elasticity problems. Gupta and Gayen [10] used multi-term Galerkin approximation in terms of Chebyshev polynomials to analyse the effect 
of two submerged unequal permeable plates in the propagation of water waves and also Roy et al. [29] used multi-term Galerkin approximation technique with Chebyshevs polynomials for water wave scattering by unequal partially immersed vertical barriers in water of uniform finite depth.

Previously, Roy et al. [27, 28,30] considered different geometrical configurations of multiple barriers in infinite depth water and formed a system of singular integral equations for each problem which were solved by using single term Galerkin expansion wherein the single-term is the exact solution of the corresponding integral equation for single barrier due to normal incidence. To avoid the complicacy of calculations using single term Galerkin approximation some researchers use Galerkin approximation in terms of simple polynomials multiplied by an appropriate weight function to solve the singular integral equations. Das et al. [4] investigated the problem of oblique scattering by thin vertical barriers in deep water by multiterm Galerkin technique using simple polynomials as basis functions. Kaur et al. [14] also used the method of polynomial approximation to solve the resulting singular integral equations for double trench problem. Recently, Das et al. [5] also used multiterm Galerkin technique with simple polynomials as basis functions to solve the integral equation arising in the problem of thick barrier in deep water.

In this paper, we have reinvestigated the problem of wave generation due to rolling of a partially immersed plate as well as a rolling submerged plate in infinitely deep water by using Galerkin approximation in terms of simple polynomials multiplied by an appropriate weight function whose form is dictated by the behaviour of the fluid velocity near the submerged edge(s) of the plate. The numerical estimate of the amplitude of the radiated wave produced by the rolling motion of the plate is obtained and it is depicted against the wave number for different sizes of the plate in a number of figures. The results given previously by Ursell [31] and Mandal [19] for partially immersed and submerged plate are recovered. Lastly, the case of swaying of the both types of plate is considered and the amplitude is obtained and depicted against the wavenumber in a number of figures and the results given by Evans [6] have been recovered.

\section{Formulation}

A Cartesian rectangular co-ordinate system is chosen in which the $y$-axis is taken vertically downwards into the fluid region and $x z$-plane is the rest position of the mean free surface $y=0$. The plate whose equilibrium position is $x=0$, $y \in L$ where $L \equiv L_{1}=(0, b)$ for partially immersed plate and $L \equiv L_{2}=(a, b)$ for submerged plate, makes small oscillations of circular frequency $\omega$ about a fixed point $(0, c)$ which need not to be in $L$. The geometry of the two positions of the plate is best described in Figure 1.

Assuming linear theory and the motion in water to be irrotational, time harmonic and independent of the co-ordinate $z$, the motion in the fluid region can be described by a velocity potential $\operatorname{Re}\left\{\phi(x, y) e^{-i \omega t}\right\}$, where $\phi$ satisfies

$$
\nabla^{2} \phi=0
$$




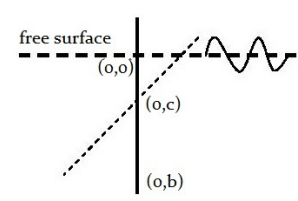

(a)

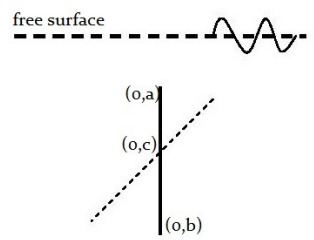

(b)

Figure 1. Diagram of rolling of (a) partially immersed plate and (b) submerged plate.

in the fluid region, the free surface condition

$$
\frac{\partial \phi}{\partial y}+K \phi=0 \text { on } y=0,-\infty<x<\infty
$$

where $K=\frac{\omega^{2}}{g}, g$ being the acceleration due to gravity, the condition due to small oscillations of the plate

$$
\frac{\partial \phi}{\partial x}=g(y) \quad \text { for } y \in L, x=0,
$$

where

$$
g(y)=-i \omega \theta_{0}(c-y)
$$

is a known function given by Evans [6] if the plate is hinged at $(0, c)$ and makes small oscillation of amplitude $\theta_{0}$. The bottom conditions for deep water is

$$
\nabla \phi \rightarrow 0 \text { as } y \rightarrow \infty, 0<x<\infty
$$

while the edge condition is

$$
r^{\frac{1}{2}} \nabla \phi \text { is bounded as } r \rightarrow 0,
$$

where $r$ is the distance from a submerged edge of the plate, and finally for $|x| \rightarrow \infty$

$$
\phi(x, y)=\left\{\begin{array}{l}
A_{-} e^{-K y-i K x} \text { as } x<0 \\
A_{+} e^{-K y+i K x} \text { as } x>0
\end{array}\right.
$$

where $A_{-}$and $A_{+}$denote the amplitude (unknown) of the water wave created by the rolling of the plate in the negative direction and positive direction of the $x$-axis respectively which is to be obtained for different sizes of the plate.

\section{Reduction to integral equation}

Use of Havelock's [12] expansion for water wave potentials produces

$$
\phi(x, y)=A_{-} e^{-K y-i K x}+\int_{0}^{\infty} A(k) S(k, y) e^{k x} d k \quad \text { for } x<0,0<y<\infty
$$


and

$$
\phi(x, y)=A_{+} e^{-K y+i K x}+\int_{0}^{\infty} B(k) S(k, y) e^{-k x} d k \quad \text { for } x>0,0<y<\infty,
$$

where $S(k, y)=k \cos k y-K \sin k y$ and $A(k)$ and $B(k)$ is an unknown functions.

The continuity of velocity at $x=0,0<y<\infty$ gives

$$
\phi_{x}(+0, y)=\phi_{x}(-0, y), \quad 0<y<\infty
$$

which gives for $0<y<\infty$

$$
i k A_{+} e^{-K y}-\int_{0}^{\infty} k B(k) S(k, y) d k=-i k A_{-} e^{-K y}-\int_{0}^{\infty} k A(k) S(k, y) d k,
$$

so that by using Havelock's inversion formula(cf. Mandal and Chakrabarti [23])

$$
\begin{aligned}
& -i K A_{-}=i K A_{+}=2 K \int_{0}^{\infty} F(u) e^{-K u} d u \\
& k A(k)=-k B(k)=\frac{2}{\pi} \frac{1}{k^{2}+K^{2}} \int_{0}^{\infty} F(u) S(k, u) d u
\end{aligned}
$$

wherein we have used

$$
\frac{\partial \phi}{\partial x}=F(y)= \begin{cases}g(y) & \text { for } y \in L, x=0 \\ f(y) & \text { for } y \in \bar{L}, x=0\end{cases}
$$

where $\bar{L} \equiv \bar{L}_{1}=(b, \infty)$ for partially immersed plate and $\bar{L} \equiv \bar{L}_{2}=(0, a) \cup$ $(b, \infty)$ for submerged plate and $f(y)$ is an unknown function having square root singularity at $y=b$ for $\bar{L}=\bar{L}_{1}$ and at $y=a, b$ for $\bar{L}=\bar{L}_{2}$.

Then, (2.1) and (3.1)-(3.3) provides

$$
\begin{aligned}
& A_{-}=-A_{+}=2 i\left[\int_{L} g(u) e^{-K u} d u+\int_{\bar{L}} f(u) e^{-K u} d u\right] \\
& A(k)=-k B(k)=\frac{2}{\pi} \frac{1}{k\left(k^{2}+K^{2}\right)}\left[\int_{L} g(u) S(k, u) d u+\int_{\bar{L}} f(u) S(k, u) d u\right] .
\end{aligned}
$$

Now the continuity of pressure at $x=0,0<y<\infty$ gives

$$
\phi(+0, y)=\phi(-0, y), \quad 0<y<\infty .
$$

This provides the integral equation for $f(y) \equiv f_{1}(y)$ for partially immersed plate given by

$$
\int_{\overline{L_{1}}} f_{1}(y) M_{1}(y, u) d u=G_{1}(y), \quad y \in \overline{L_{1}}
$$

where

$M_{1}(y, u)=2 i e^{-K(y+u)}-\frac{1}{\pi} \ln \left|\frac{y-u}{y+u}\right|-\frac{2}{\pi} e^{-K(y+u)} \int_{-\infty}^{K(y+u)} \frac{e^{v}}{v} d v, \quad y, u \in \overline{L_{1}}$ 
and $\forall y \in \overline{L_{1}}$

$$
G_{1}(y)=\int_{L_{1}} g(u)\left[\frac{1}{\pi} \ln \left|\frac{y-u}{y+u}\right|+\frac{2}{\pi} e^{-K(y+u)} \int_{-\infty}^{K(y+u)} \frac{e^{v}}{v} d v-2 i e^{-K(y+u)}\right] d u,
$$

while

$$
f_{1}(y)=O\left((y-b)^{-\frac{1}{2}}\right) \text { as } y \rightarrow b+0 .
$$

The integral equation for $f(y) \equiv f_{2}(y)$ for submerged plate is given by

$$
\int_{\overline{L_{2}}} f_{2}(y) M_{2}(y, u) d u=G_{2}(y), \quad y \in \overline{L_{2}},
$$

where

$M_{2}(y, u)=2 i e^{-K(y+u)}-\frac{1}{\pi} \ln \left|\frac{y-u}{y+u}\right|-\frac{2}{\pi} e^{-K(y+u)} \int_{-\infty}^{K(y+u)} \frac{e^{v}}{v} d v, \quad y, u \in \overline{L_{2}}$

and

$G_{2}(y)=\int_{L_{2}} g(u)\left[\frac{1}{\pi} \ln \left|\frac{y-u}{y+u}\right|+\frac{2}{\pi} e^{-K(y+u)} \int_{-\infty}^{K(y+u)} \frac{e^{v}}{v} d v-2 i e^{-K(y+u)}\right] d u$ $y \in \overline{L_{2}}$,

while

$$
f_{2}(y)=\left\{\begin{array}{l}
O\left((a-y)^{-\frac{1}{2}}\right) \text { as } y \rightarrow a-0 \\
O\left((y-b)^{-\frac{1}{2}}\right) \text { as } y \rightarrow b+0
\end{array}\right.
$$

It may be noted that the integral equations (3.4) and (3.5) are weakly singular integral equations. These integral equations were solved in the literature in closed from using somewhat complicated procedure. However, the complicated procedure for each barrier configuration is avoided here and the integral equations are solved numerically using Galerkin approximation involving simple polynomials multiplied by appropriate weight functions whose form is dictated by the edge condition. Very accurate numerical estimates for the amplitude of the radiated waves are obtained from the approximate solution of the integral equations.

\section{Numerical solutions of the integral equations}

Numerical solutions of the integral equations (3.4) and (3.5) are now obtained by using Galerkin approximation in terms of simple polynomials multiplied by weight functions whose forms are dictated by the appropriate edge conditions. Thus we expand $f_{1}(y)$ and $f_{2}(y)$ as

$$
f_{1}(y)=\left(\frac{b}{b-y}\right)^{\frac{1}{2}} e^{-K y} \sum_{n=0}^{N} a_{n}\left(\frac{y}{b}\right)^{n} \quad \text { for } b<y<\infty
$$


and

$$
f_{2}(y)= \begin{cases}\left(\frac{a}{a-y}\right)^{\frac{1}{2}} \sum_{n=0}^{N} b_{n}\left(\frac{y}{a}\right)^{n} & \text { for } 0<y<a, \\ \left(\frac{b}{y-b}\right)^{\frac{1}{2}} e^{-K y} \sum_{n=0}^{N} c_{n}\left(\frac{y}{b}\right)^{n} & \text { for } b<y<\infty,\end{cases}
$$

where $N$ is an integer to be chosen suitably.

Substituting the expression of $f_{1}(y)$ given by (4.1), $f_{2}(y)$ given by (4.2) in place of $f(y)$ and $g(y)$ given by (2.2) in (3.3) we obtain $A_{-}$and $A_{+}$as given by,

$$
\begin{aligned}
& A_{-}=-A_{+} \\
& =\left\{\begin{array}{c}
2 i\left[-\int_{0}^{b} i \omega \theta_{0}(c-u) e^{-K u} d u+\sum_{n=0}^{N} a_{n} \int_{b}^{\infty}\left(\frac{b}{b-u}\right)^{\frac{1}{2}}\left(\frac{u}{b}\right)^{n} e^{-2 K u} d u\right] \\
\text { for partially immersed plate, } \\
2 i\left[-\int_{a}^{b} i \omega \theta_{0}(c-u) e^{-K u} d u+\sum_{n=0}^{N} b_{n} \int_{0}^{a}\left(\frac{a}{a-u}\right)^{\frac{1}{2}}\left(\frac{u}{a}\right)^{n} e^{-K u} d u\right. \\
\left.+\sum_{n=0}^{N} c_{n} \int_{b}^{\infty}\left(\frac{b}{u-b}\right)^{\frac{1}{2}}\left(\frac{u}{b}\right)^{n} e^{-2 K u} d u\right] \quad \text { for submerged plate. }
\end{array}\right.
\end{aligned}
$$

To find the unknown constants $a_{n}(n=0,1,2, \ldots, N)$, we put $y=\frac{b}{y_{i}}(i=$ $0,1,2, \ldots, N),\left(0<\frac{b}{y_{i}}<1\right)$ in the relation (3.4) to obtain the linear system

$$
\begin{aligned}
& \sum_{n=0}^{N} a_{n} A_{i n}=G\left(\frac{b}{y_{i}}\right), i=0,1,2, \ldots, N, \\
& A_{\text {in }}=\int_{b}^{\infty}\left(\frac{b}{b-u}\right)^{\frac{1}{2}}\left(\frac{u}{b}\right)^{n} e^{-K u} M_{1}\left(\frac{b}{y_{i}}, u\right) d u, \quad i=0,1,2, \ldots, N .
\end{aligned}
$$

Suitable transformations like $u=\frac{b}{w}$ can be made to change the range of the integration to a finite one. The collocation points $\frac{b}{y_{i}}$ are to be chosen suitably. Here we have chosen

$$
b / y_{i}=1-i / N, \quad i=0,1,2, \ldots, N .
$$

Actually $i=0$ or $N$ have not been used. For $i=0$ or $N$ a point little bit away from end points (towards right for $i=0$ and towards left for $i=N$ ) have been chosen during the numerical computation to avoid singularity.

To find the unknown constants $b_{n}$ and $c_{n}(n=0,1,2, \ldots, N)$ we put

$$
y=\left\{\begin{array}{lr}
y_{i}(i=0,1,2, \ldots, N) & \text { for } 0<y_{i}<a, \\
b / y_{i}(i=0,1,2, \ldots, N) & \text { for } 0<\frac{b}{y_{i}}<1
\end{array}\right.
$$

to obtain the combined linear system

$$
\begin{aligned}
& \sum_{n=0}^{N} b_{n} B_{i n}+\sum_{n=0}^{N} c_{n} C_{i n}=G\left(y_{i}\right), \quad i=0,1,2, \ldots, N, \\
& \sum_{n=0}^{N} b_{n} B_{i n}+\sum_{n=0}^{N} c_{n} C_{i n}=G\left(\frac{b}{y_{i}}\right), \quad i=0,1,2, \ldots, N,
\end{aligned}
$$


where

$$
\begin{aligned}
B_{\text {in }} & =\int_{0}^{a}\left(\frac{a}{a-u}\right)^{\frac{1}{2}}\left(\frac{u}{a}\right)^{n} M_{2}\left(y_{i}, u\right) d u, \quad i=0,1,2, \ldots, N, \\
C_{\text {in }} & =\int_{b}^{\infty}\left(\frac{b}{u-b}\right)^{\frac{1}{2}}\left(\frac{u}{b}\right)^{n} e^{-K u} M_{2}\left(\frac{b}{y_{i}}, u\right) d u, \quad i=0,1,2, \ldots, N .
\end{aligned}
$$

To find $C_{i n}(i=0,1,2, \ldots, N)$, suitable transformations like $u=\frac{b}{w}$ can be made to change the range of the integration to a finite one. The collocation points are to be chosen as

$$
\begin{aligned}
& y_{i}=i a / N \text { for } 0<y_{i}<a, i=0,1,2, \ldots, N, \\
& b / y_{i}=1-i / N \text { for } 0<b / y_{i}<1, \quad i=0,1,2, \ldots, N .
\end{aligned}
$$

Here also, $i=0$ or $N$ have not been used. For $i=0$ or $N$ a point little bit away from end points (towards right for $i=0$ and towards left for $i=N$ ) have been chosen during the numerical computation to avoid singularity.

Now, the linear systems (4.3), (4.4) and (4.5) can be solved by any standard method to obtain the constants $a_{n}, b_{n}$ and $c_{n}(n=0,1,2, \ldots, N)$. Hence the approximate solution of the integral equation (3.4) and (3.5) are obtained.

\section{Special cases}

In the case of swaying of a partially immersed and submerged plate given by Evans [6] and Mandal [19], that is when $\theta_{0} \longrightarrow 0$ and $c \longrightarrow \infty$ in such a way that $\theta_{0} c=\Gamma$ is a finite real constant called the amplitude of sway (cf. Evans [6]), then the expression of $g(y)$ given by (2.2) becomes

$$
g^{*}(y)=-i \omega \Gamma
$$

and the amplitude of the radiated wave of a swaying plate is then obtained.

\section{$5 \quad$ Numerical results}

\subsection{Convergence of the numerical results}

To study the convergence of the numerical results on $N(N+1$ is the number of terms in the polynomial approximation), numerical estimates for $\left|A^{*}\right|\left(=\left|\frac{A}{\omega \theta_{0}}\right|\right.$ as in [18], $\left.|A|=\left|A_{+}\right|=\left|A_{-}\right|\right)$is tabulated in Tables 1 and 2 for the partially immersed and submerged plates respectively for $N=1,2, \ldots, 5$ and different values of wave number $K b$ and other parameters mentioned therein. Table 1 shows the number of terms of the simple polynomial in the Galerkin approximation given by $N=1,2, \ldots, 5$. The value of the amplitude converges up to three or four decimal places for the partially immersed plate as computed from the exact expression given by Ursell [31] for $c / b=0.4$. Similarly, Table 2 shows the number of terms of the simple polynomial in the Galerkin approximation given by $N=1,2, \ldots, 5$. The value of the amplitude converges up to three or four decimal places for the partially immersed plate as computed from the exact expression given by Mandal [19] for $a / b=0.2$ and $c / b=0.5$. Thus for all numerical computations $N=4$ is chosen throughout. 
Table 1. Values of $\left|A^{*}\right|$ against $K b$ for different values of $N$ for a partially immersed plate with $c / b=0.4$.

\begin{tabular}{|c|c|c|c|c|c|c|}
\hline$K b$ & $\begin{array}{c}\text { Ursell } \\
(1947)\end{array}$ & $\begin{array}{c}\left|A^{*}\right| \\
(N=1)\end{array}$ & $\begin{array}{c}\left|A^{*}\right| \\
(N=2)\end{array}$ & $\begin{array}{c}\left|A^{*}\right| \\
(N=3)\end{array}$ & $\begin{array}{c}\left|A^{*}\right| \\
(N=4)\end{array}$ & $\begin{array}{c}\left|A^{*}\right| \\
(N=5)\end{array}$ \\
\hline 0.30 & 0.0044968 & 0.0042668 & 0.004328 & 0.0043568 & 0.0044712 & 0.0044774 \\
\hline 0.45 & 0.0005737 & 0.0004967 & 0.0004973 & 0.0005343 & 0.00055745 & 0.0005634 \\
\hline 0.60 & 0.0113109 & 0.0110232 & 0.0112766 & 0.0113365 & 0.0113023 & 0.0113219 \\
\hline 0.75 & 0.0242648 & 0.0249897 & 0.0246533 & 0.0243655 & 0.0242751 & 0.0242688 \\
\hline 0.90 & 0.035554 & 0.0354981 & 0.0355198 & 0.0355378 & 0.0355117 & 0.0355294 \\
\hline 1.05 & 0.0442971 & 0.0443568 & 0.0443414 & 0.04424351 & 0.0442543 & 0.0442658 \\
\hline 1.20 & 0.0511054 & 0.0511126 & 0.0511047 & 0.0511086 & 0.0511091 & 0.0511035 \\
\hline 1.35 & 0.0566166 & 0.0567652 & 0.056734 & 0.0566128 & 0.0566354 & 0.0566187 \\
\hline 1.50 & 0.0612313 & 0.0611874 & 0.0612645 & 0.0612726 & 0.0612944 & 0.0612388 \\
\hline
\end{tabular}

Table 2. Values of $\left|A^{*}\right|$ against $K b$ for different $N$ for a submerged plate with $a / b=0.2$ and $c / b=0.5$.

\begin{tabular}{ccccccc}
\hline$K b$ & $\begin{array}{c}\text { Mandal } \\
(1991)\end{array}$ & $\begin{array}{c}\left|A^{*}\right| \\
(N=1)\end{array}$ & $\begin{array}{c}\left|A^{*}\right| \\
(N=2)\end{array}$ & $\begin{array}{c}\left|A^{*}\right| \\
(N=3)\end{array}$ & $\begin{array}{c}\left|A^{*}\right| \\
(N=4)\end{array}$ & $\begin{array}{c}\left|A^{*}\right| \\
(N=5)\end{array}$ \\
\hline 0.31 & 0.0209182 & 0.0204345 & 0.0207587 & 0.0209654 & 0.0209764 & 0.0209134 \\
0.51 & 0.0392564 & 0.0391747 & 0.0391158 & 0.0391941 & 0.0392747 & 0.0392843 \\
0.71 & 0.0471074 & 0.0473679 & 0.0471288 & 0.0471105 & 0.0471064 & 0.0471087 \\
0.91 & 0.0491133 & 0.0496542 & 0.0492761 & 0.0491545 & 0.0491087 & 0.0491241 \\
1.11 & 0.0477695 & 0.0478254 & 0.0476429 & 0.0477635 & 0.0477583 & 0.0477847 \\
1.31 & 0.0446953 & 0.0445788 & 0.0447277 & 0.0446105 & 0.0446235 & 0.0446876 \\
1.51 & 0.0400838 & 0.0401852 & 0.0401208 & 0.0400965 & 0.0400557 & 0.0400896 \\
1.71 & 0.0322326 & 0.0322175 & 0.0322658 & 0.0322759 & 0.0322484 & 0.0322398 \\
1.91 & 0.0217085 & 0.0217652 & 0.0217275 & 0.0217118 & 0.0217077 & 0.0217064 \\
\hline
\end{tabular}

\subsection{Graphical representation of the numerical results}

In Figure 2, we have plotted $\left|A^{*}\right|$ for the rolling motion of a partially immersed plate for $c / b=0.4$ against $K b$. The curve totally coincides with the curve drawn from the explicit solutions of the amplitude at infinity given by Ursell [31]. In Figure 3 , we have plotted $\left|A^{*}\right|$ for the rolling motion of the submerged plate for $c / b=0.5$ and $a / b=0.2$ against $K b$. This curve totally coincides with the curve drawn from the explicit expression given by Mandal [19]. These two figures provide a check on the correctness of the method employed here.

In Figure $4,\left|A^{*}\right|$ is depicted against $K b$ for a submerged plate taking $a / b=$ $0.0001,0.01,0.05,0.1$ and $c / b=0.5$. When $a / b=0.0001$, the value of $\left|A^{*}\right|$ is almost zero when $K b$ is zero and then increases with the wave number $K b$. For the other values of $a / b=0.01,0.05,0.1$, the amplitude increases at first and then decreases nearly to zero for some value of $K b$ and then again increases with $K b$ for larger values of $a / b$.

In Figure $5,\left|A^{*}\right|$ is depicted against $K b$ for a submerged plate for different values of $a / b=0.1,0.15,0.2,0.25$ and $c / b=0.5$. Here, the value of the amplitude starts from zero and rapidly increases until it reaches a peak and then 
decreases upto zero at some point and again increases as $K b$ increases further, thus showing a oscillatory behaviour.

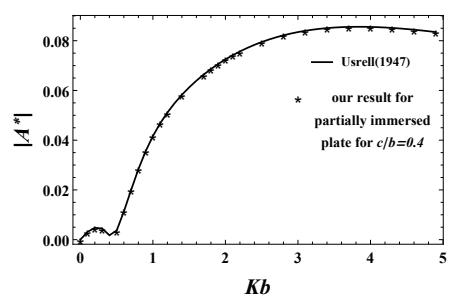

Figure 2. $\left|A^{*}\right|$ vs $K b$ for partially immersed plate for $c / b=0.4$.

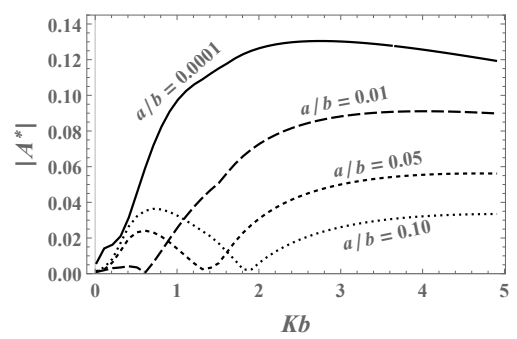

Figure 4. $\left|A^{*}\right|$ vs $K b$ for $a / b=0.0001$ to $a / b=0.1$ and $c / b=0.5$.

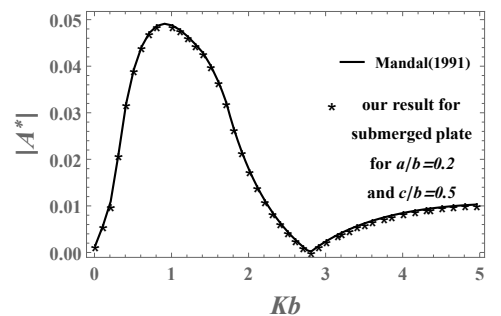

Figure 3. $\left|A^{*}\right|$ vs $K b$ for submerged plate for $c / b=0.5$ and $a / b=0.2$.

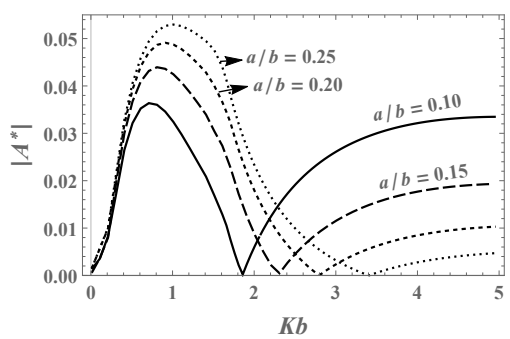

Figure 5. $\left|A^{*}\right|$ vs $K b$ for $a / b=0.1$ to $a / b=0.25$ and $c / b=0.5$.

In Figure 6, $\left|A^{*}\right|$ for a submerged plate is plotted against $K b$ for $a / b=$ $0.25,0.35,0.45$ and $c / b=0.5$. From this figure it is observed that, as $a / b$ increases $\left|A^{*}\right|$ first increases upto a peak value as a function of the wave number $K b$ and then decreases asymptotically to zero as $K b$ further increases.

Figure 7 depicts $\left|A^{*}\right|$ for a submerged plate when the point $(0, c)$ lies above the plate i.e. the plate undergoes a small rolling oscillations about a point which lies above it. We choose $a / b=0.6,0.7,0.8,0.95$ and $c / b=0.5$. Here also the same nature of the amplitude can be observed as before but the peak value of $\left|A^{*}\right|$ is smaller when larger values of $a / b$ are considered. Here also the value of the amplitude asymptotically tends to zero for higher values of $K b$.

The difference of change of $\left|A^{*}\right|$ can also be observed for the different values of the oscillatory point $c / b$ keeping $a / b$ fixed. In Figure $8,\left|A^{*}\right|$ is depicted against $K b$ for $c / b=0.5,0.6,0.7$ and $a / b=0.2$. Here the oscillatory behaviour of the amplitude is observed and in Figure $9,\left|A^{*}\right|$ is depicted for the same set of values of $c / b$ with fixed $a / b=0.45$. Different peak points for $\left|A^{*}\right|$ can be observed for different values of $c / b$ and for all cases $\left|A^{*}\right|$ becomes small as $K b$ increases further.

Figure 10 depicts $\left|A^{*}\right|$ for the case of swaying of a partially immersed plate. The curve for $\left|A^{*}\right|$ almost coincides with the same drawn from the expression 
given by Evans [6] considering the value of the constant $\Gamma=\frac{1}{\omega \pi}$. It is observed that $\left|A^{*}\right|$ is zero when the value of $K b$ is zero and then $\left|A^{*}\right|$ increases while $K b$ increases and then decreases after reaching e peak value as $K b$ further increases. The coincidence of the numerical values of the amplitude of a swaying partially immersed plate with Evans [6] gives another check on the correctness of the method employed here.

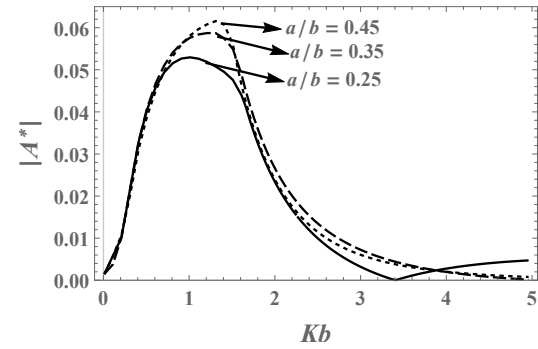

Figure 6. $\left|A^{*}\right|$ vs $K b$ for $a / b=0.25$ to $a / b=0.45$ and $c / b=0.5$.

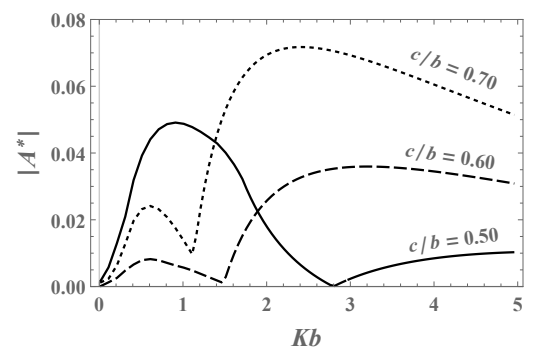

Figure 8. $\left|A^{*}\right|$ vs $K b$ for $a / b=0.2$ for different values of $c / b$.

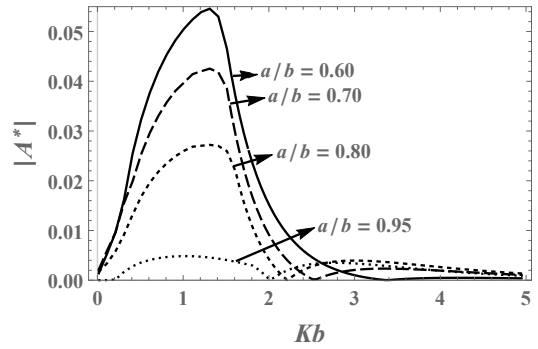

Figure 7. $\left|A^{*}\right|$ vs $K b$ for $a / b=0.6$ to $a / b=0.95$ and $c / b=0.5$.

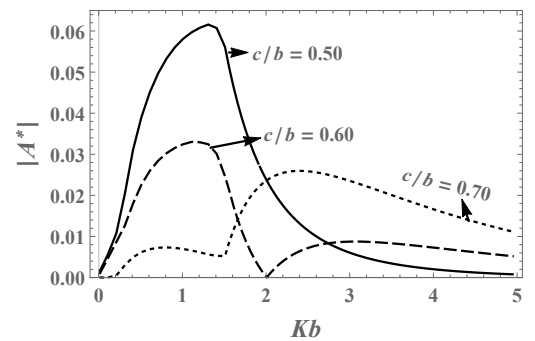

Figure 9. $\left|A^{*}\right|$ vs $K b$ for $a / b=0.45$ for different values of $c / b$.

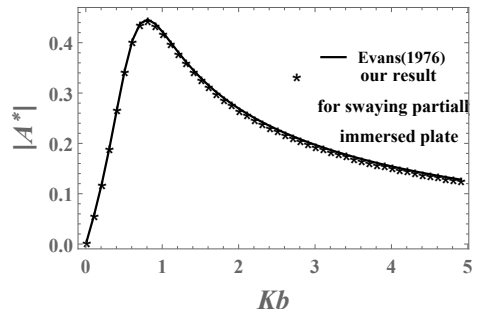

Figure 10. $\left|A^{*}\right|$ vs $K b$ coincides with Evans [6] for a swaying partially immersed plate.

The amplitude of a swaying submerged plate can similarly be observed in Figures 11 and 12 for $a / b=0.0001,0.05,0.2,0,4$ and for $a / b=0.5,0.6,0.7,0.9$ respectively. Here also the amplitude becomes higher at first and then begin to decrease along with the increase of $K b$ and tends to zero for further increase 
of $K b$. It is also observed that for $a / b=0.9$ i.e. when the size of the plate is very much small the amplitude becomes almost zero, which is obvious.

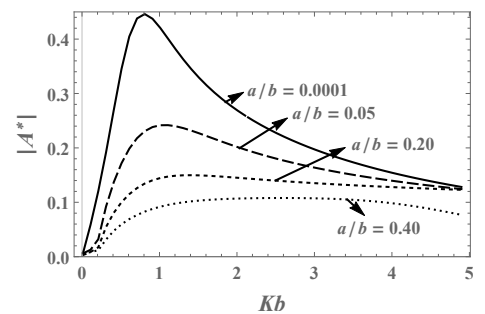

Figure 11. $\left|A^{*}\right|$ vs $K b$ for $a / b=0.0001$ to $a / b=0.4$ for swaying plate.

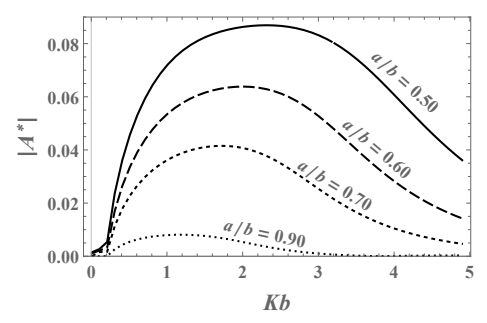

Figure 12. $|A|$ vs $K b$ for $a / b=0.5$ to $a / b=0.9$ for swaying plate.

\section{Conclusions}

Here the problems of surface water wave generated by rolling oscillations of a thin vertical plate partially immersed as well as submerged in deep water are reinvestigated. The integral equation for the horizontal component of velocity along the plate is solved numerically by Galerkin technique wherein the unknown functions are expanded in terms of simple polynomials multiplied by an weight function whose form is dictated by the edge condition of the plate. Very accurate numerical estimates for the amplitude of the radiated waves are obtained and are depicted in a number of figures against the wave number. The effect of change of the size of the submerged plate has been studied for rolling as well as swaying of the plates.

It may be noted that, in some water wave problems involving thin plates the original integral equations to which these are reduced are logarithmically singular (weakly singular). Then after some mathematical operations involving differentiation (once or twice) these are reduced to strongly singular (Cauchy singular or hypersingular) integral equations. Fortunately, in some cases the strongly singular integral equations (usually Cauchy singular) can be solved in closed forms. However, this may not succeed always, for example for the case of thin curved plates. In those cases, one has to employ some appropriate numerical methods. As most of these methods involve expansion involving some special functions (which are basically polynomials), the polynomial approximation employed here is also applicable. That Cauchy singular and hypersingular integral equations can be solved numerically by using polynomial approximations can be seen in the papers by Mandal and Bera $([21,22])$.

\section{Acknowledgements}

The authors thank the reviewers for their comments and suggestions to revise the paper in the present form. SR thanks CSIR (File No. 09/028(1018)/2017EMR-I), New Delhi, for providing financial assistance. This work is also supported by SERB, New Delhi, through the research project EMR/2016/005315. 


\section{References}

[1] S. Banerjea, D.P. Dolai and B.N. Mandal. On waves due to rolling of a ship in water of finite depth. Q. J. Mech. Appl. Math., 67:35-43, 1996. https://doi.org/10.1007/BF00787137.

[2] S. Banerjea and B.N. Mandal. On waves due to rolling of a vertical plate. Indian J. Pure Appl. Math., 23:753-761, 1992.

[3] H. Cheng, M.J. Peng and Y.M. Cheng. The hybrid complex variable elementfree Galerkin method for 3D elasticity problems. Engg. Structures, 219:110835, 2020. https://doi.org/10.1016/j.engstruct.2020.110835.

[4] B.C. Das, S. De and B.N. Mandal. Oblique scattering by thin vertical barriers in deep water: solution by multiterm Galerkin technique using simple polynomials as basis. J. Marine Sc. Tech., 23:915-925, 2018. https://doi.org/10.1007/s00773017-0520-4.

[5] B.C. Das, S.De and B.N. Mandal. Oblique water waves scattering by a thick barrier with rectangular cross section in deep water. J. Engg. Math., 2020. https://doi.org/10.1007/s/0665-020-10049-4.

[6] D.V. Evans. A note on the waves produced by the small oscillations of a partially immersed vertical plate. J. Inst. Maths. Applics., 17:135-140, 1976. https://doi.org/10.1093/imamat/17.2.135.

[7] D.V. Evans and M. Fernyhough. Edge waves along periodic coastlines. Part 2. Mathl. Comput. Modelling, 297:307-325, 1995. https://doi.org/10.1017/S0022112095003119.

[8] D.V. Evans and C.A.N. Morris. The effect of a fixed vertical barrier on obliquely incident surface waves in deep water. J. Inst. Math. Appl, 9:198-204, 1972. https://doi.org/10.1093/imamat/9.2.198.

[9] D.V. Evans and R. Porter. Hydrodynamic characteristics of a thin rolling plate in finite depth of water. Appl. Ocean Res., 18(4):215-228, 1996. https://doi.org/10.1016/S0141-1187(96)00026-0.

[10] S. Gupta and R. Gayen. Water wave interaction with dual asymmetric non-uniform permeable plates using integral equations. Appl. Math. Comp., 346(1):436-451, 2019. https://doi.org/10.1016/j.amc.2018.10.062.

[11] M.D. Haskind. Radiation and diffraction of surface waves by a flat plate floating vertically. Prikl. Mat. Mech, 23(3):546-556, 1959. https://doi.org/10.1016/00218928(59)90168-6. (In Russian).

[12] T.H. Havelock. Forced surface waves on water. Phillos. Mag, 8:569-576, 1929. https://doi.org/10.1080/14786441008564913.

[13] M. Kanoria and B.N. Mandal. Water wave scattering by a submerged circular-arc-shaped plate. Fluid Dyn. Res., 31(5):317-331, 2002. https://doi.org/10.1016/S0169-5983(02)00136-3.

[14] A. Kaur, S.C. Martha and A. Chakrabarti. Solution of the problem of propagation of water waves over a pair of asymmetrical rectangular trenches. Appl. Ocean Res, 93(101946), 2019. https://doi.org/10.1016/j.apor.2019.101946.

[15] A.-J. Li, Y. Liu and H.-J. Li. Accurate solutions to water wave scattering by vertical thin porous barriers. Mathematical Problems in Engineering, 2015(985731), 2015. https://doi.org/10.1155/2015/985731. 
[16] F.B. Liu and Y.M. Cheng. The improved element-free Galerkin method based on the nonsingular weight functions for inhomogeneous swelling of polymer gels. Int. J. Appl. Mech., 10(4):1850047, 2018. https://doi.org/10.1142/S1758825118500473.

[17] F.B. Liu, Q. Wu and Y.M. Cheng. A meshless method based on the nonsingular weight functions for elastoplastic large deformation problems. Int. J. Appl. Mech., 11(1):1950006, 2019. https://doi.org/10.1142/S1758825119500066.

[18] P. Maiti, P. Rakshit and S. Banerjea. Wave motion in an ice covered ocean due to small oscillations of a submerged thin vertical plate. J. Marine Sc. Appl., 14:355-365, 2015. https://doi.org/10.1007/s11804-015-1326-6.

[19] B.N. Mandal. On waves due to small oscillations of a vertical plate submerged in deep water. J. Austral. Math. Soc. Ser., 32(3):296-303, 1991. https://doi.org/10.1017/S0334270000006871.

[20] B.N. Mandal, S. Banerjea and M. Kanoria. The rolling ship problem-revisited. Mathl. Comput. Modelling, 25(1):11-18, 1997. https://doi.org/10.1016/S08957177(96)00181-1.

[21] B.N. Mandal and G.H. Bera. Approximate solution for a class of hypersingular integral equations. Appl. Math. Letter, 19(11):1286-1290, 2006. https://doi.org/10.1016/j.aml.2006.01.013.

[22] B.N. Mandal and G.H. Bera. Approximate solution of a class of singular integral equations of second kind. J. Comp. Appl. Math., 206(1):189-195, 2007. https://doi.org/10.1016/j.cam.2006.06.011.

[23] B.N. Mandal and A. Chakrabarti. Water wave scattering by barriers. WIT Press, Southampton, 2000.

[24] Z.J. Meng, H. Cheng, L.D. Ma and Y.M. Cheng. The hybrid elementfree Galerkin method for threedimensional wave propagation problems. International Journal for Numerical Methods in Engineering, 117(1):15-37, 2018. https://doi.org/10.1002/nme.5944.

[25] R. Porter and D.V. Evans. Complementary approximations to wave scattering by vertical barriers. J Fluid Mech., 294:155-180, 1995. https://doi.org/10.1017/S0022112095002849.

[26] P. Rakshit and S. Banerjea. Effect of bottom undulation on the waves generated due to rolling of a plate. J. Marine Sc. Tech., 10:7-16, 2011. https://doi.org/10.1007/s11804-011-1035-8.

[27] R. Roy, U. Basu and B.N. Mandal. Water-wave scattering by two submerged thin vertical unequal plates. Arch. Appl. Mech., 86:1681-1692, 2016. https://doi.org/10.1007/s00419-016-1143-7.

[28] R. Roy, U. Basu and B.N. Mandal. Water wave scattering by a pair of thin vertical barriers with submerged gaps. J. Engg. Math., 105:85-97, 2017. https://doi.org/10.1007/s10665-016-9884-4.

[29] R. Roy, S. De and B.N. Mandal. Water wave scattering by multiple thin vertical barriers. Appl. Math. Comp., 355:458-481, 2019. https://doi.org/10.1016/j.amc.2019.03.004.

[30] R. Roy and B.N. Mandal. Water wave scattering by three thin vertical barriers with middle one partially immersed and outer two submerged. Meccanica, 54:7184, 2019. https://doi.org/10.1007/s11012-018-0922-3.

[31] F. Ursell. On waves due to rolling motion of a ship. Q. J. Mech. Appl. Math., 1:246-252, 1947. https://doi.org/10.1093/qjmam/1.1.246. 